\title{
Wave induced stress profile on a paired column semisubmersible hull formation for column reinforcement
}

\author{
Agbomerie Charles Odijie, Stephen Quayle and Jianqiao Ye* \\ Engineering Department, Lancaster University, Lancaster, LA1 4YW, United Kingdom
}

\begin{abstract}
A study into reinforcing the hull of the recently developed paired column semisubmersible platform has been carried out by understanding the stress profile around its columns from hydrodynamic interaction during survival and extreme weather conditions in the Gulf of Mexico. The conceptualization of this hull system is to enable dry-tree technology on semisubmersibles for deep-sea exploration. Its hydrodynamic response behaviour has been confirmed to be compatible with this technology, although its size and high steel requirement are of major disadvantage. Preliminary CFD study has showed an unusual flow behaviour within and around the hull due to its unique column arrangement. This behaviour creates an unusual hydrodynamic pressure profile on the hull, dominated by the wave parameters. Numerical models were developed using ANSYS and AQWA to compute the stress distribution on the columns from this unique uneven hydrodynamic pressure. The boundary conditions for the FE-model were formulated using hydrostatic stiffness theories and hydrodynamic response plots developed in Orcaflex. The results have showed high stress concentration on the inner columns. For operating conditions (low wave amplitude), the wave propagating direction was observed to have little or no effect on the column stress distribution. Significant effect of the wave propagating angle was observed as its amplitude gradually increases. Results for topside and deck mass effect on the stress distribution on the columns also suggested high stress distribution around the joint area of the inner columns for extreme and survival weather conditions, irrespective of the flow orientation.
\end{abstract}

Keywords: PC-Semi, Stress distribution, Deep-draft, Finite element analysis

\section{Nomenclature}

$\begin{array}{ll}\text { Symbols } & \\ \phi & \text { Velocity potential } \\ P_{h y d} & \text { Hydrodynamic pressure } \\ g & \text { Gravity } \\ \rho & \text { Sea water density } \\ A_{w} & \text { Wave amplitude } \\ \theta & \text { Wave propagating angle } \\ \lambda & \text { Wave length } \\ \varepsilon & \text { Perturbation parameter } \\ \nabla & \text { Laplace grad operator } \\ H_{S} & \text { Wave height } \\ \mathrm{z} & \text { Draft height }\end{array}$


$\mathrm{h}$

$\psi_{i}$

$\phi_{\text {ir }}$

$\phi_{R}$

$s_{w}$

$s_{w}^{a v g}$

$\sigma_{e}$
Sea depth

Radiation unit amplitude

Radiation unit velocity potential

Radiation potential

Wetted surface

Average area of wetted surface

Equivalent stress (von Mises stress)

\section{Abbreviations}

PC-Semi

ABS

DNV

CFD

RAO

MODU

FPI

$\mathrm{RP}$

DOF

API

RPSEA

FSI
Paired Column Semisubmersible

American Bureau of Shipping

Det Norske Veritas

Computational Fluid Dynamics

Response Amplitude Operator

Mobile Offshore Drilling Unit

Floating Production Installation

Recommended Practice

Degrees of Freedom

American Petroleum Institute

Research Partnership to Secure energy for America

Fluid Structure Interaction

\subsection{Introduction}

\subsection{Overview}

Column-stabilized semisubmersibles are the most used type of semisubmersible in ocean engineering. The design employs the use of vertical columns to suspend a superstructure from the waterline, and supported at the bottom with a pontoon system. Deep draft semisubmersible hulls systems are mainly used for designing drilling and production units in the oil and gas industry. The recently developed paired column semisubmersible (Pc-Semi) platform for dry tree application has added to the fleet. This newly developed hull system has been reported to have unique motion behaviour in deep-sea, which makes it favourable for deep water production platform design. The uniqueness of its dynamic behaviour has been observed in its wave influenced and vortex-induced motions. Different reports have been presented as an attestation to that fact [[14]]. However, the influence of the wave behaviour on the deformation and stress distribution on the hull has not been studied. The hydrodynamic interactions from wave, current and wind loads sometimes make it difficult to access the strength of large floating bodies.

A literature review was carried out first to understand how wave energy is transferred on floating structures. The theoretical background of this was discussed in [5] in a review of hydro-elastic theories for response of marine structures. This review explained in a broad view the development of two and three dimensional (linear and nonlinear) theories used in evaluating fluid structural interactions of deformable parts in marine structures. The text presented in [6] gave a detailed understanding on the motions and deformation experienced in barges 
and columns under regular wave loading, assuming that the flow was incompressible, inviscid, non-rotational and of small wave amplitude. With these assumptions, mathematical models were generated to calculate the bending and continuous deformation at different mode shapes of a free-free (both ends) submerge floating column and barge. In resolving the hydrodynamics of a single slender vertical column, high resonant deflection was noticed within the range of occurring wave energy. Comparisons for hinge joints and free conditions were analysed for the problem of a floating barge, and the effect of constraints was investigated for certain degrees of freedom[7] described the hydrodynamic nature of fluid interactions between a set of arranged columns supporting a large flexible structure (pontoon). He resolved the motion equations of the topside integral part considering the effect the trapped wave and flow circulation within the columns, and the possible effects of stress and elastic deflection of these on the topside. Solutions of this problem require dealing with complex issues such as, flow separation and boundary layer shear. Diffraction analysis is the most effective way to resolve this problem (evaluating the wave effect on floating structures), without having to deal with the above mentioned complex issues. [8]made progressive contributions in resolving the diffraction problems experienced with multiple floating structures in directional waves using finite element method. Their analysis was focused on structural response investigation in regular wave with small amplitude, and they were able to make significant conclusions on the relationship between force and response ratio criteria. Although the results of their analysis might not have a wide range of application in real life scenarios, its detail presentation served as a significant prerequisite for this study. [9]studied the fluid-structure interaction (FSI) of three-dimensional bodies in water waves and were able to develop an interaction theory that effectively predicted the diffraction characteristics of each member of the body.

Early studies on PC-Semi showed that the column plate deformation was due to a number of factors that actually varied with wave height (Hs) and its orientation. Results recorded in [10] highlighted some factors e.g., column shape, second moment of area, drag force coefficient, draft size and plate thickness that are most likely to alter the extent to which the column plates will deform from hydrodynamic loadings in rough weather. From the systematic literature review, alongside a preliminary CFD study, the unique arrangement of columns was observed to create flow circulation within the hull structure which in turn generates an uneven drag around the columns. The wave also experiences an asymmetric relationship in the space within the eight columns, creating an uneven stress profile around them, which is greatly influenced by the uneven hydrodynamic pressure distribution that is exerted from wave-current interactions. These circulations coupled with the wave loads create an unusual loading on the columns which results in deformation of the hull. The strength of floating hulls is determined from their buckling tendencies and stress distribution, providing information on where necessary reinforcement were required.

This paper introduces a research study on how column reinforcement can be carried out on a paired column semisubmersible platform subjected to the stress profile from the wave loads specified in DNV and ABS standards. In this study, we described the nature and effect of wave-induced stress profile on the columns of a PC-Semi, using a finite element approach. The study also provides a detailed analysis on the factors influencing the uneven hydrodynamic force parameters on the hull of a PC-Semi. The wave load and boundary conditions used for formulating the FE-model were extracted from hydrodynamic models developed in AQWA and Orcaflex. At the end of this study, areas requiring high steel reinforcement are identified, to guarantee the safety of the hull under twisting and bending phenomenon.

\subsection{Hull Description}

A typical PC-Semi is made of eight rectangular columns, (four inner columns and four outer columns), a topside, pontoon, inner-outer column connections, and inner column braces. The inner columns are slightly smaller than the outer ones, to reduce the oscillating amplitude of the shed vortexes during high current velocities [11]. Figure 1 shows the geometry adopted in this study, which was extracted from [12]. The inner and outer columns are $14.0 \mathrm{~m} \times 10.40 \mathrm{~m}$ and $14.0 \mathrm{~m} \times 13.40 \mathrm{~m}$ respectively. The edges of the columns were designed with a fillet curve of $2.01 \mathrm{~m}$ radius, which reduced the respective diagonal distance of the columns to $17.72 \mathrm{~m}$ and $15.60 \mathrm{~m}$. This reductions alter the effect of vortex shedding phenomenon on this hull, details of which will be presented in subsequent sections. The curved edges also help to distribute the stresses around the plates. The columns on each pair are $20.4 \mathrm{~m}$ apart from their base. 


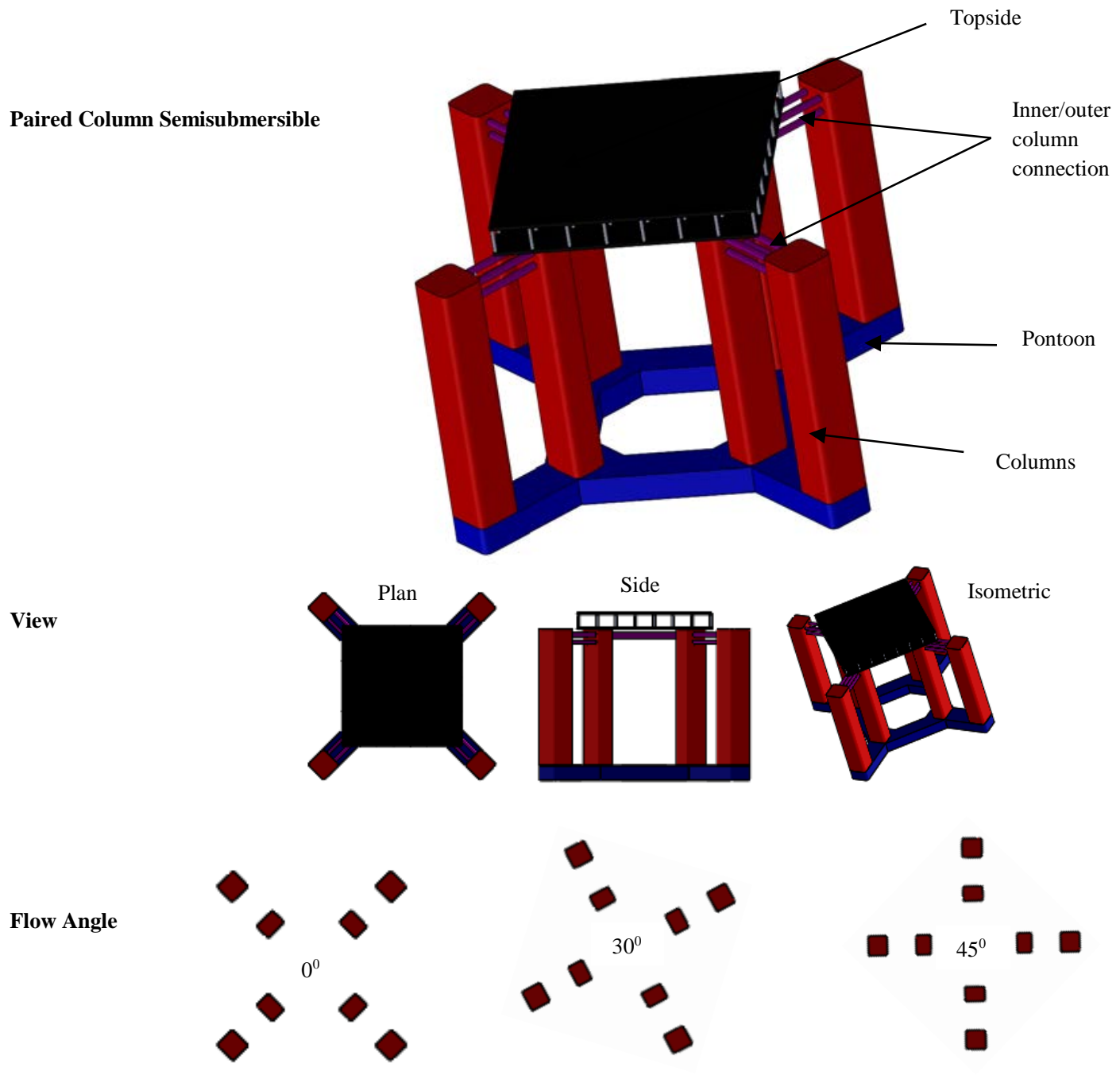

Figure 1 Hull geometry

\subsection{Theory Formation}

\subsection{Hydrodynamics}

For large floating bodies such as a PC-Semi hull, a good understanding of the diffracted and radiation wave conditions are required to describe the dynamic effect of the fluid structure interactions on the strength and stability of the columns. Hydrodynamic correlations were used to estimate the flow pressure and force parameters in this study. The correlations are based on the resolution of fluid potential of a free floating body with six degrees of freedom. For an irrotational, incompressible and inviscid fluid, considering an impermeable hull, the velocity field (flow domain) satisfies the Laplace expression in Equation 1

$$
\nabla^{2} \phi=0
$$

Where $\nabla$ is the Laplace operator and $\phi$ is the velocity potential. In a rectangular coordinate system, the above equation becomes

$$
\nabla^{2} \phi=\left(\partial^{2} \phi\right) /\left(\partial x^{2}\right)+\left(\partial^{2} \phi\right) /\left(\partial y^{2}\right)+\left(\partial^{2} \phi\right) /\left(\partial z^{2}\right)=0
$$

Equation 2 is solved after considering the boundary conditions at the free surface, seafloor and any submerged surface of the hull, e.g., at the seafloor 


$$
\partial \phi / \partial z=0
$$

Bernoulli's equation relates the hydrodynamic pressure $\left(P_{h d y}\right)$ to a flow stream and its velocity potential as;

$$
P_{h d y}=-\rho[\partial \phi / \partial t+(1 / 2) \nabla \phi * \nabla \phi+g h]
$$

Where $\rho$ is the density of seawater, and $h$ is considered as the height of the submerged area of the hull (Draft height), $\mathrm{t}$ denotes time.

Different wave theories can be applied in resolving the complexities associated with the resolving velocity potential around the boundaries defined in the equations above. Linear regular wave theory (Airy wave) expresses the total wave potential as a summation of the incident, diffraction and radiation wave potentials. (Equation 5)

$$
\phi=\phi_{I}+\phi_{S}+\phi_{R}
$$

Where $\phi_{I}$ is the incident wave potential, $\phi_{s}$ is the scattered wave potential, and $\phi_{R}$ is the radiation potential.

One major challenge with this method is the boundary value problem associated with the radiation potential, which is due to the motion of the body after impact. The total radiation potential $\left(\phi_{R}\right)$ is the last term in Equation 5 that can be expressed in terms of the motion amplitude (Equation 6), where $\psi_{i}$ is the unit radiation amplitude in each degree of freedom, and $\phi_{i r}$ is the unit radiation potential in each degree of freedom.

$$
\phi_{R}=\sum_{i=1}^{6} \psi_{i} \phi_{i r}
$$

After solving the equation (2), [13] expressed the velocity potential from linear wave theory in its complex form as;

$$
\phi=-\left[\left(i g A_{w} \cosh [k(z+h)]\right) / \omega \cosh (k h)\right] e^{(i(k x \cos \theta+k y \sin \theta-\omega t))}
$$

Where $A_{w}$ is the wave amplitude, $z$ is the sea depth, $\omega$ is the wave frequency, $\theta$ is the propagating direction of the wave, $\mathrm{t}$ is the time, and $\mathrm{k}$ is the wave number measured in relation with the wave length $\lambda$ as $2 \pi / \lambda$.

Equation 7 is only used in scenarios where linear waves are considered. Ocean waves are known for their irregular behaviour. For this case, the correlations postulated from Stokes $2^{\text {nd }}$ wave is adopted. The wave is considered to progressively change from a steady to an unsteady state. Its unsteady nature is computed using perturbation formulation and expressed in Taylor's series as

$$
\phi=\phi^{(0)}+\phi^{(1)}+\phi^{(2)}+0\left(\varepsilon^{3}\right)
$$

Using this theory, the velocity potential is derived as

$$
\phi=-\left(3 \omega A_{w}^{2} \cosh [2 k(z+h)]\right) /\left(8 \sinh ^{4} \mathrm{kh}\right) e^{(i(k x \cos \theta+k y \sin \theta-\omega t))}
$$

Hydrodynamic force on the hull was therefore obtained by integrating the hydrodynamic pressure around its wetted surface (Equation. 10)

$$
F_{h y d}=-\int_{s_{w}}^{\dot{p}} p n_{j} d s
$$


The hydrodynamic pressure is obtained by substituting Equations 7 and 9 into Equation 4, for regular and irregular flow, respectively. The motion of the body is relative to wetted surface area of the submerged part of the hull $\left(s_{w}\right)$, as the hull oscillates the submerged area/surface changes, and also experiences a level of deformation $\delta$. The force on the wetted surface is therefore estimated from the summation of the pressure due to the average wetted surface area $s_{w}^{a v g}$, and the change created due to the motion $\partial s_{w}^{a v g}$

$$
F_{h y d}=-\rho g \iint_{s_{w}} p n_{j} d s=-\rho g\left[\iint_{s_{w}} p n_{j} d s+\iint_{\delta s_{w}^{a v g}} p n_{j} d s\right]
$$

\subsection{Load and Element Description}

The columns are hollow structures. The internal space is often partitioned into different sections, and used for liquid storage (ballast liquid, drilling mud, dead oil). The stresses on the columns are therefore due to the internal and external forces. In this study, we have considered only the external forces, as the standard for designing semisubmersible hull in deep waters recommends only the external loadings. Figure 2 shows schematic representations of the external loads on the columns. The weld connections at the top and bottom of the columns were modelled differently. A direct interface was used between the topside and inner columns, while the hydrostatic stiffness matrix was used for the connection between the columns and the pontoon. Details of this boundary formulation is discussed at the later part of the paper. Five external loads sources were considered, as shown in Figure 2. Table 1 presents a description of the five loads. Apart from the topside and the pontoon, the columns also have braces connection attached to them, as shown in Figure 2. The hull was therefore modelled using shell, 3D-solid, contact, surface, and target elements. SHELL181 was used for columns and pontoon plates, while SURF154 was for surface ocean loading. The braces, inner/outer column connections, fairleads and topside trusses, were built with SOLID187, while CONTA173 and TARGE170 were used to define the contact between the bodies to take their deformation into account. Shell181 (S4 in ABAQUS) was preferred to other elements because of its six DOF at each node which represents the actual case of a floating structure subject to not only surge, sway and heave, but also roll, pitch and yaw. It has a unique quality of easy convergence during simulation and allows easy identification of the effect of pressure distribution. The effect of plate thickness can easily be studied because it allows alteration during coding.

Table 1 Column load definition

\begin{tabular}{l|l}
\hline No. & Load description \\
\hline 1 & Topside weight \\
2 & Buoyancy force from displaced volume \\
3 & Force from flow pressure due to current velocity \\
4 & Wave forces, relative to wave amplitude \\
5 & Forces from flow shear and interactions; vortex formations \\
\hline
\end{tabular}



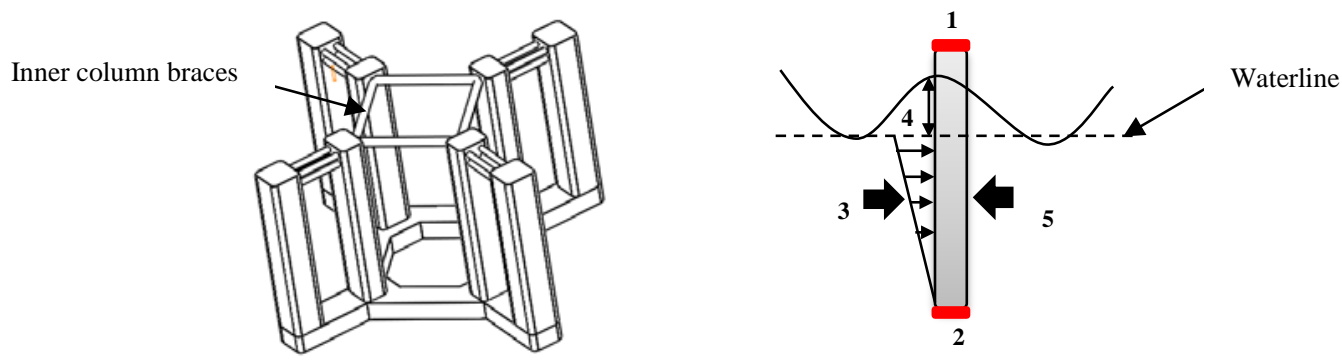

Figure 2 Description of loads on column

SHELL181 is a 4-node 3-D element with six degrees of freedom. Its formation is based on the fundamental thin-wall shell theories recorded in [14-17]. Some researchers have employed the use of this type of element to analyse the behaviour of plate structures exposed to ocean loadings. [18] used a 4-node shell element (S4) to investigate the buckling failure mode of semisubmersible columns under ocean loadings. The numerical set-up was developed in ABACUS, alongside an experimental study. With the element, they were able to effectively study the buckling mode of a stiffened semisubmersible column. [19] also made use of elements developed from thin-wall shell theories to investigate the hydro-elastic behaviour of ships. Shell elements generally have a unique quality of easy convergence during simulation and allows easy identification of the effect of pressure distribution. The effect of plate thickness can easily be studied because it allows alteration during coding. SOLID187 is advantageous for an irregular grid (mesh), as in the case of this study where the geometric complexity was from the inclusion of the connections, braces, topside-beams, and fairleads. Further description and functionalities of this element can be found in ANSYS release 17 [20].CONTA173 is a 3D element with 3 degrees of freedom. It was used to define the surface contact between the solid and shell elements, i.e., between shell-to-shell contact, shell-to-beam contact, and beam-to-beam contact. Figure 3 shows the areas around the hull where the contact elements applies. The contact elements were represented by TARGE170 as shown in Figure 3. It also shows surface of shell-to-beam contact; columns and topside / connections. Quadratic triangular meshes were employed around these regions. The regions are shell-to-shell contacts; columns and pontoon. Linear quadrilateral meshes were used on these surfaces.

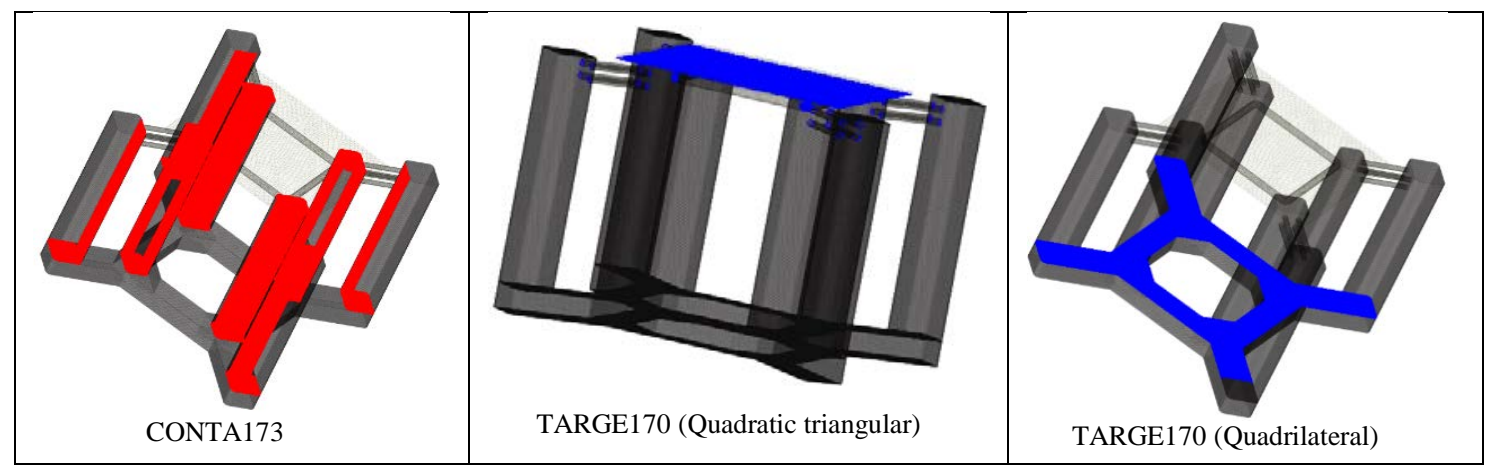

Figure 3 Element location

The finite element model for the shell element was derived using fundamentals shell theories. The response of the nodes and surface were analysed in natural coordinates ' $r_{i}$ ' and Cartesian coordinate $x_{i}, y_{i}$ and $z_{i}$. 


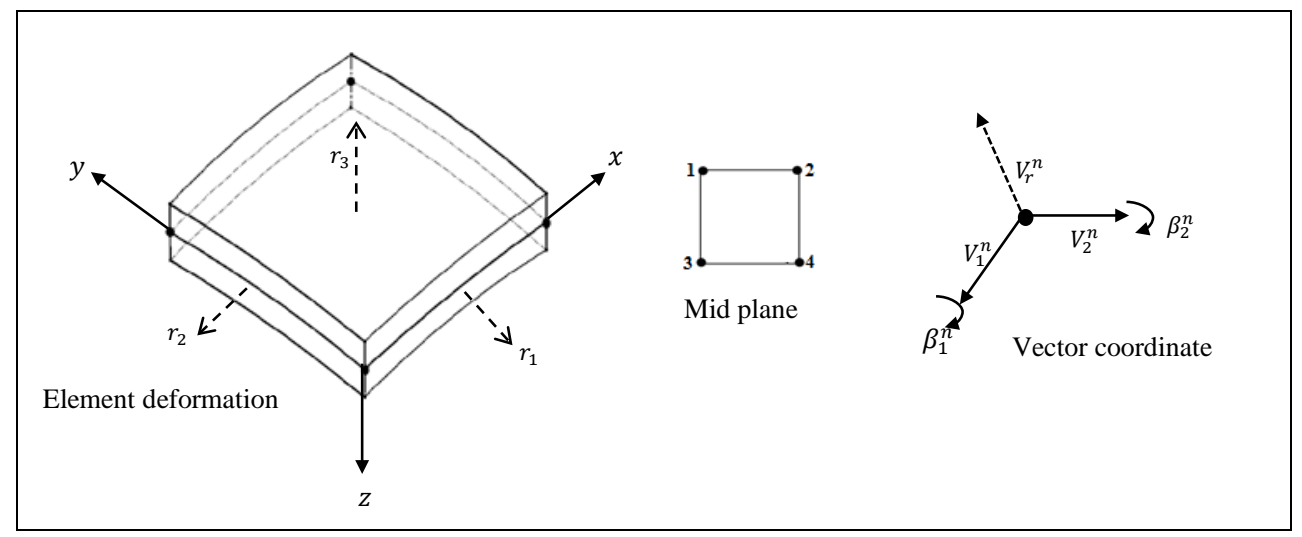

Figure 4 Element description

The element has four nodes and 6 degrees of freedom as shown in Figure 4 above. The two-dimensional vector components are denoted by $V_{1}^{n}$ and $V_{2}^{n}$ and let their rotational components be $\beta_{1}^{n}$ and $\beta_{2}^{n}$. From the element kinetics, the surface geometry in Cartesian coordinate for the ' $x$ ' coordinate for ' $i$ ' mode of vibration can be written as

$$
x_{i}=\sum_{n=1}^{4} h_{n} x_{i}^{n}+\frac{r_{3}}{2} \sum_{n=1}^{4} t_{n} h_{n} V_{r i}^{n}
$$

Where $t_{n}$ is the thickness of the shell section at each node which is normally measured along the resultant vector ' $V_{r i}^{n}$ ' of $V_{1}$ and $V_{2}$.

The displacement $u_{i}$ of the element surface considering the normal coordinates is

$$
u_{i}=\sum_{n=1}^{4} h_{n} u_{i}^{n}+\frac{r_{3}}{2} \sum_{n=1}^{4} t_{n} h_{n}\left(-V_{2 i}^{n} \beta_{1}^{n}+V_{1 i}^{n} \beta_{1}^{n}\right)
$$

\subsection{Numerical Procedure}

The purpose of this research is to study the elastic and stress behaviour of the hull described in Section 2 and identify areas around the columns where high reinforcement is required. It will also aid the material selection procedure during scantling. The numerical procedure was developed in two phases; hydrodynamic or diffraction analysis and finite element analysis. In carrying out hydrodynamic analysis, the loading and boundary conditions imposes challenges such as fluid separation, radiation, viscosity. Diffraction helps to simplify the effect of these challenges [7], and some other theories have been also developed over the years to help circumvent the effect of these challenges. Equations for hydrodynamic diffraction can be found in most text on floating structures, and a complex form of the wave properties have been described in Section 2 of this paper.

\subsection{Model for Hydrodynamic (diffraction) Analysis}

The hydrodynamic diffraction analysis was developed in ANSYS AQWA (Release 16) for specific sea and weather conditions. The hull model for this analysis was selected from the already designed paired column semis as seen in Table 2. Free floating conditions were considered as shown in Figure 5 (without the effect of topside, moorings, risers and other structural attachments), and results for impact pressure at different flow directions were recorded. The pressure and motion results resolved from the diffraction analysis were used as ocean loadings for the finite element analysis. Emphasis was placed on investigating the hydrodynamic pressure forces acting on each element and node for various flow orientations using a time series analysis. 


\begin{tabular}{lc}
\hline Hull & Dimensions \\
\hline Draft size & $53.34 \mathrm{~m}$ \\
Exposed column & $28.04 \mathrm{~m}$ \\
Submerdged column & 45.42 \\
Inner column size & $10.4 \mathrm{~m} \times 14 \mathrm{~m}$ \\
Outer column size & $13.4 \mathrm{~m} \times 14 \mathrm{~m}$ \\
Inner column span & $50.3 \mathrm{~m}$ \\
Outer column span & $95.98 \mathrm{~m}$ \\
Plate thickness & $0.0508 \mathrm{~m}$ \\
Sea depth & $2400 \mathrm{~m}$ \\
\hline
\end{tabular}

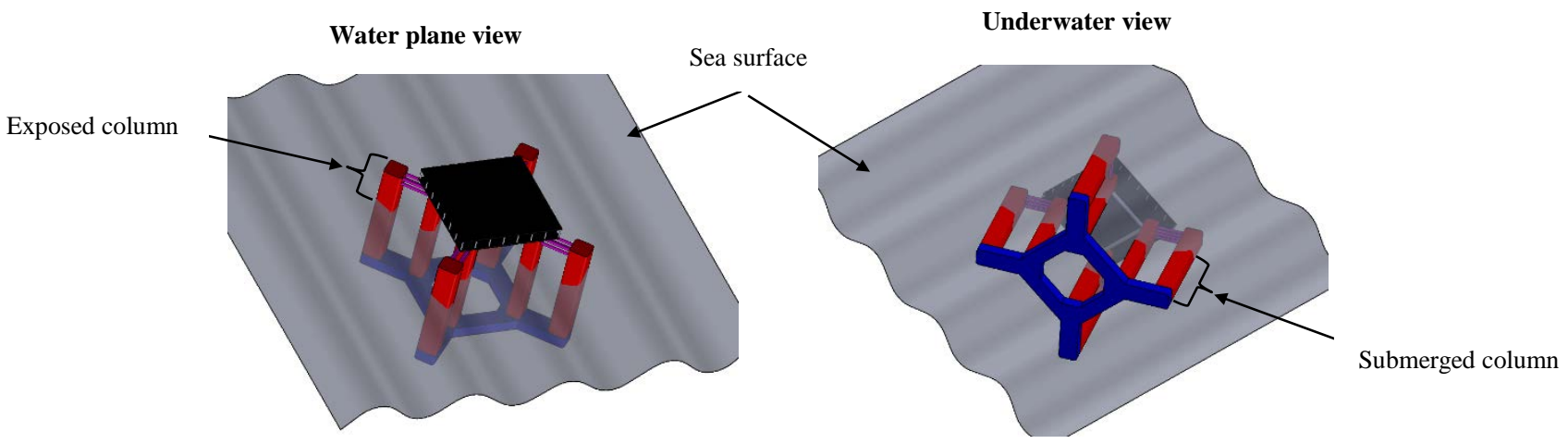

Figure 5 Ocean view

Mesh independence study was carried out on the hull model designed for hydrodynamic analysis to help increase the results accuracy. For the effectiveness of mess density and tolerance, the element size was varied between $2 \mathrm{~m}$ and $1.15 \mathrm{~m}$ and the effects on the maximum RAO at $0^{0}$ incidences in the $\mathrm{Z}$ direction were recorded. The results in Table 3 show no significant variation in the RAO value for heave motion at $0^{0}$ flow angle for the same range of wave frequencies. Figure 6 shows the hydrodynamic mesh with element size 1.15. It is important to mention that no basic refinement was made on any particular section of the hull and straight edges were considered for mesh uniformity.

Table 3 Grid independence for diffraction analysis (Heave study)

\begin{tabular}{|c|c|c|c|}
\hline $\begin{array}{c}\text { Element } \\
\text { size (m) }\end{array}$ & $\begin{array}{c}\text { No. of } \\
\text { elements }\end{array}$ & $\begin{array}{c}\text { No. of } \\
\text { nodes }\end{array}$ & $\begin{array}{c}\text { Max. RAO } \\
\mathbf{( m / m )}\end{array}$ \\
\hline 2.0 & 8948 & 9058 & 1.747 \\
\hline 1.8 & 11252 & 11374 & 1.738 \\
\hline 1.6 & 13925 & 14061 & 1.740 \\
\hline 1.4 & 18061 & 18215 & 1.736 \\
\hline 1.2 & 24055 & 24237 & 1.731 \\
\hline 1.15 & 25861 & 26045 & 1.711 \\
\hline
\end{tabular}




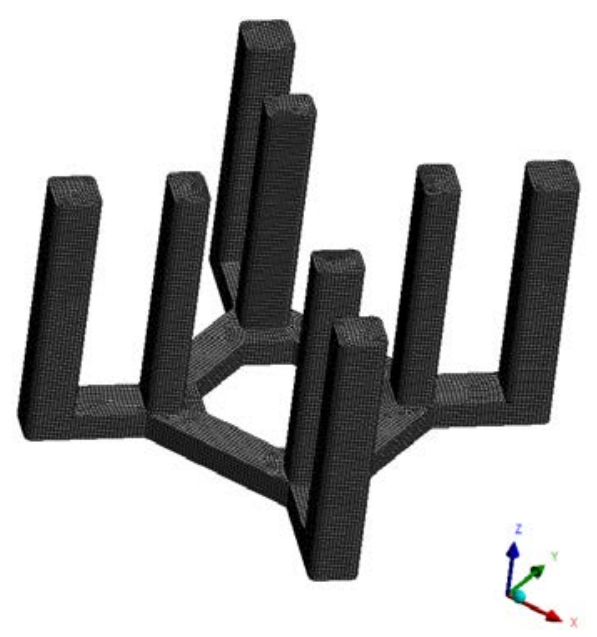

Figure 6 Hydrodynamic model

\subsection{Ocean and Load Conditions for Hydrodynamic Model}

Recommended standards for environmental loading conditions on offshore structures include [21],[22, 23]. Different regions of the same ocean (Atlantic) recorded different weather conditions. For the Gulf of Mexico, [21] presented conditions for west (between $97.5^{\circ}$ and $95^{\circ}$ ), west-central (between $94^{\circ}$ and $90.5^{\circ}$ ), central (between $89.5^{\circ}$ and $86.5^{\circ}$ ) and east (between $85.5^{\circ}$ and $82.5^{\circ}$ ). The variation between the recorded values played a significant role in our selection process for estimating the weather conditions adopted in this study. Conventional designs are studied for different load conditions depending on what standard is adopted. The conditions include survival, extreme, severe, damage, tow, accident, and operating. In this study, our design was tested for two conditions; survival and extreme conditions. Our decision was based on the conclusion made from the literature review and company reports that maximum values for structural response were recorded for these two cases. Table 4 shows the wave conditions adopted for this study. Values for survival conditions are the wave data for 1000years return period while the extreme conditions are wave data for 100years return period, as recommended by [22].

Table 4 Environmental case study

\begin{tabular}{l|c|c}
\hline Parameters & Survival condition & Extreme condition \\
\hline Significant wave height (m) & 16.4 & 13.1 \\
Maximum wave height (m) & 30.1 & 24.2 \\
Peak spectral period (s) & 16.7 & 15.1 \\
Period of maximum wave (s) & 15.1 & 13.6 \\
\hline
\end{tabular}

A typical offshore structure has multiple hydrodynamic load sources, but for assessing strength and fatigue behaviour, the most critical loading scenarios are investigated in this paper. Acceptable loading conditions for assessing the strength of offshore platforms are presented in the literature and industrial standards. The loading system is addressed in their static and dynamic phases. [24] presented generally acceptable loading conditions for Mobile Offshore Units (MODU) and Floating Production Installations (FPI). The static and combined loadings are basically required for MODU, while the normal operation and severe storm scenarios are investigated for FPI; as presented in Table 5. The dynamic loads are as a result of environmental conditions (wave, current and wind), while the static loads are defined with the uneven mass distribution around the structure.

Table 5 DNV standard for loading conditions for MODU and FPI

\begin{tabular}{l|l|l|l}
\hline \multicolumn{2}{c|}{ Static } & \multicolumn{1}{c|}{ Combined } & \multicolumn{2}{c}{ Normal operation } & \multicolumn{2}{c}{ Severe storm } \\
\hline $\begin{array}{l}\text { Forces to be } \\
\text { considered include }\end{array}$ & $\begin{array}{l}\text { The inclusion of } \\
\text { environmental }\end{array}$ & $\begin{array}{l}\text { Considering of all } \\
\text { working conditions; }\end{array}$ & $\begin{array}{l}\text { Loadings from harsh } \\
\text { or rough weather }\end{array}$ \\
\hline
\end{tabular}




\begin{tabular}{|c|c|c|c|}
\hline $\begin{array}{l}\text { the mass of hulls } \\
\text { structure and the } \\
\text { operational loads. No } \\
\text { water motion is } \\
\text { considered. }\end{array}$ & $\begin{array}{l}\text { loadings to static } \\
\text { loadings. }\end{array}$ & $\begin{array}{l}\text { inclusion of all } \\
\text { possible topside loads, } \\
\text { live load, dead loads } \\
\text { and all other operating } \\
\text { loads. }\end{array}$ & $\begin{array}{l}\text { conditions. } 100 \text { and } \\
1000 \text { wave return } \\
\text { period red } \\
\begin{array}{l}\text { considered in this } \\
\text { case. }\end{array}\end{array}$ \\
\hline
\end{tabular}

In this study, we have considered combined, normal operation and severe storm under 'dynamic load' conditions. Our analysis were carried for the worst possible sea conditions, for 100years and 1000years wave return periods.

The mass calibration was carried out considering two reference points; the centre of gravity and the buoyancy centre. The gravity centre is where the downward forces were computed from and the centre of buoyancy is where the upward thrust is calculated from. The masses are categorized into five groups;

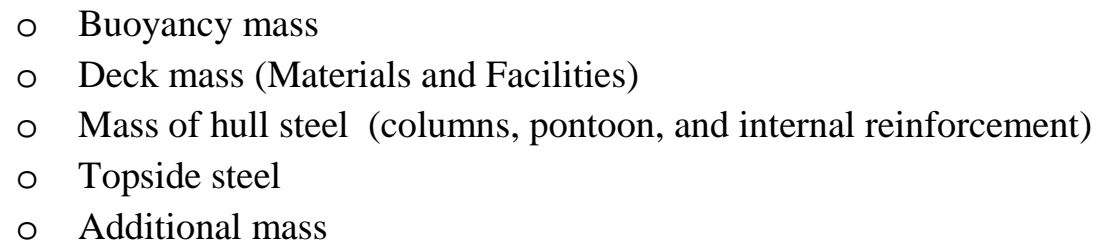

It is important to mention that at equilibrium (no ocean loading), the summation of the reaction forces from the mass distribution equals zero at the cut water plane, i.e., the mass of the entire structure equals the mass of the displaced volume of water (Archimedes principle). The mass of the displaced volume of water is regarded as the buoyancy mass, from which the upward buoyancy force is calculated. In this study we assumed that the weight on the deck (platform) is always evenly distributed. This might not necessarily be the case during operation as the staff working on-board might move equipment around.

From the view of practical design, the error margin that might result from this is negligible. A study into existing semisubmersibles was carried out to be able to effectively estimate the total amount deck mass for this study. The drilling rig and the house quarters are usually the heavy components associated with the deck mass. Recently developed semisubmersibles used for drilling purpose were designed for high payload. For example, Ocean Apex and Ocean Baroness were designed to accommodate 140 and 138 people, respectively. The mass of the hull steel is the mass of steel required to build/fabricate the columns, braces, welds, and pontoon section. This involves the amount of steel plate and internal reinforcement (scantling) used. It is important to note that the liquid content within the hollow section of the hull (such as blast liquid and dead oil) are not considered. The weight of the deck structure was estimated to be between $5.2 \times 10^{6} \mathrm{Kg}$ and $6.3 \mathrm{x}$ $10^{6} \mathrm{Kg}$. This estimation was done from the deck type selected. In this study, other masses outside the above mentioned masses was classified under additional mass. Materials, such as the ballast liquid and riser liquid are considered as also additional mass. The value for additional mass was varied, depending on the functionality the hull was designed for. Table 6 shows the summary of the mass calibration used in this study.

Table 6 Summary of Mass Calibration

\begin{tabular}{l|c|c}
\hline Mass & Component & Magnitude (Kg) \\
\hline Buoyancy & Mass of the displaced water & $96.5 \times 10^{6}$ \\
Deck & Facilities and utilities & $(25.1$ to 34$) \times 10^{6}$ \\
Hull steel & Columns, pontoon, braces \& internal & $26.72 \times 10^{6}$ \\
& reinforcement & \\
Topside steel & Truss and plate & $9.1 \times 10^{6}$ \\
Additional & Ballast liquid, dead oil, & 4.5 to $11.0 \times 10^{6}$ \\
\hline
\end{tabular}




\subsection{Finite Element Model (Load and Boundary Formulation)}

For the finite element modelling, the hydrodynamic loads are transferred as pressure loads and RAOs. The pressure calculated at each node on the hydrodynamic model are transferred into the nodes in the FE-model. This requires the columns and pontoon mesh type used for hydrodynamic analysis to be the same as for the FE-analysis. One major challenge encountered in carrying out this load transfer is defining the support conditions at both ends of the columns. The stress distribution and load concentration on the columns are controlled by the nature of boundary conditions around the hull. Developing the accurate boundary conditions for structural analysis of a semi-submerged free floating structure can be very tricky as there is no basic form of constraint on any part around the structure (see Figure 5) apart from the added mass and damping effect on the submerged part section, and the loadings around the structural component is not the same (static and dynamic cases). The outer columns have no direct contact with the topside (in some cases), while the inner columns have direct loadings from the topside in all cases. The elastic behaviour of the hull is therefore controlled by the nature of the hydrostatic boundary conditions around the hull, exerted by the flow. The static effect of the fluid on the submerged section of the hull restricts its behaviour on the vertical plane. The ocean loads cuts through the columns $28.04 \mathrm{~m}$ from the top and the wave forces are distributed around the structure from this plane. The motion in the 6 degrees of freedom and the stiffness in each of these directions are therefore considered to be the factors responsible for controlling the boundary. We considered the translational and rotational displacement components for dynamic load cases. The reason for this was that the response analysis showed movements from the cut-water plane and the hull base. The displacement parameters were computed using time series method in Orcaflex, and the results were recorded over a specific time period. The data was arranged in a tabular form and used as boundary conditions during the build-up of the numerical model. This form of boundary condition involves motions in all 6DOF, and this replicates the actual scenario of the structure in its dynamic state. This method is not time efficient. A hydrostatic stiffness matrix was employed to circumvent the challenges associated with the displacement/rotation boundary method. The hydrostatic stiffness at the cut water plane shows the resultant relationship between the structural load and the weight generated from its displacement (also known as buoyancy) to the motion characteristics (heave, roll and pitch) from the centre plane to which all the forces act. It is derived from the early analytical formulation of the equation of motions governing a single degree of freedom system. The derived stiffness is made of a 3 $\mathrm{X} 3$ matrix after eliminating the zero elements from a $6 \mathrm{X} 6$ matrix recorded in the surge, sway and yaw. It is important to mention that the hydrostatic stiffness of a free floating body is zero on it horizontal plane. This is why the motions operating on this plane (X, Y and RZ) have zero components. Table 7 shows the hydrostatic stiffness on the cut-water plane for heave, roll and pitch DOF.

Table 7 Cut-Water Plane Stiffness

\begin{tabular}{|c|c|c|c|}
\hline & Heave & Roll & Pitch \\
\hline & \multicolumn{3}{|c|}{ Cut- Water Plane } \\
\hline Heave & \multirow{3}{*}{$\begin{array}{c}12977894 \mathrm{~N} / \mathrm{m} \\
- \\
1.9918276 \mathrm{~N} . \mathrm{m} / \mathrm{m} \\
- \\
42.757839 \mathrm{~N} . \mathrm{m} / \mathrm{m}\end{array}$} & \multirow{3}{*}{$\begin{array}{c}-0.034764 \mathrm{~N} /{ }^{0} \\
180024000 \mathrm{~N} \cdot \mathrm{m} /{ }^{0} \\
52.457371 \mathrm{~N} \cdot \mathrm{m} /{ }^{0}\end{array}$} & \multirow{2}{*}{$\begin{array}{c}-0.7288118 \mathrm{~N} /{ }^{0} \\
52.457371 \mathrm{~N} . \mathrm{m} /{ }^{0}\end{array}$} \\
\hline Roll & & & \\
\hline \multirow[t]{2}{*}{ Pitch } & & & $180024000 \mathrm{~N} . \mathrm{m} /{ }^{0}$ \\
\hline & \multicolumn{3}{|c|}{ Heel of Columns } \\
\hline Heave & $12977894 \mathrm{~N} / \mathrm{m}$ & $-0.034764 \mathrm{~N} /{ }^{0}$ & $12977894 \mathrm{~N} / \mathrm{m}$ \\
\hline Roll & $\begin{array}{c}- \\
1.9918276 \mathrm{~N} . \mathrm{m} / \mathrm{m}\end{array}$ & $569193000 \mathrm{~N} \cdot \mathrm{m} /{ }^{0}$ & $\begin{array}{c}- \\
1.9918276 \mathrm{~N} . \mathrm{m} / \mathrm{m}\end{array}$ \\
\hline Pitch & 42.757839N.m/m & $52.457371 \mathrm{~N} \cdot \mathrm{m} /{ }^{0}$ & $569193000 \mathrm{~N} . \mathrm{m} /{ }^{0}$ \\
\hline
\end{tabular}


Previous researchers have faced complex challenges in coupling this stiffness matrix with the structural matrix during the modelling procedure. The reason is because the fluid has no direct contact with the area generating its hydrostatic behaviour on this plane. This creates complexities during FE-analysis. For stress analysis, [25] modelled the cut-water plane stiffness of a paired column semisubmersible hull and took it as the plan stiffness at the base of the columns. This is a much conserved approach in resolving this problem, because from the hydrostatic theory, the static effect of the fluid on the hull around the base of the columns is different to that at the cut-water plane. In this study, we calculated the hydrostatic stiffness at the base of the columns, and applied it as the boundary condition at that location. This helped to increase the accuracy of the results. We compared the results obtained using this method and the displacement method (for column analysis), and an excellent agreement between these scenarios was observed. Clearly, the advantage of using this method is that it takes less computational time when compared with the displacement method.

\subsection{Materials}

The offshore industry recommends between grades 50 to 80 steel for constructing drilling and production semisubmersible hull systems. The yield stress of the recommended ranges between 350MPa to 550MPa. Maximum plate thickness for hull construction is 2inches $(0.0508 \mathrm{~m})$. The density of steel is $7850 \mathrm{Kg} / \mathrm{m}^{3}$, with a modules of 201GPa.

\subsection{Results and discussion}

The stress distribution on the column was studied for different weather conditions, topsides, column edges, flow angles, deck weight, and plate thickness. Figure 7 shows an illustration of the resultant effect of wave loadings on a paired column semisubmersible hull using nodal contour plot of hydrodynamics and stress distribution around the columns. It is evident that the plates 'do not' experience the same deformation as they are located at different positions in the flow stream. This creates a variation of stress profiles on each edge at the top and bottom. It is important to mention that it was observed that the wave period ' $T$ ' had not any significant effect on the maximum stress recorded for the same wave heights occurring at different periods.

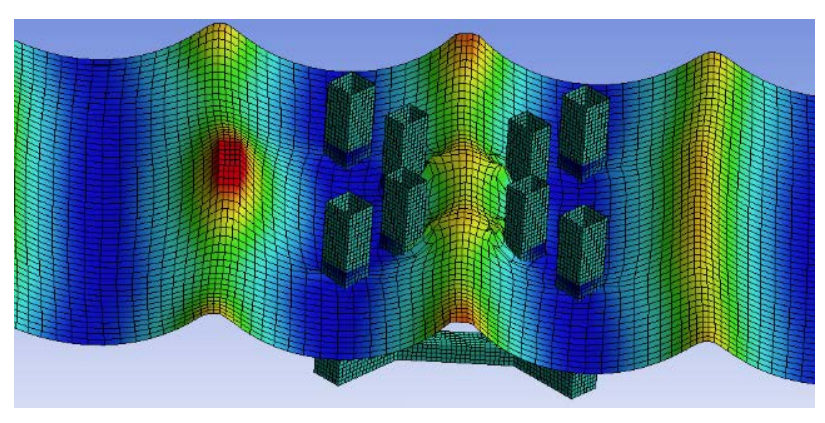

Hydrodynamic loading

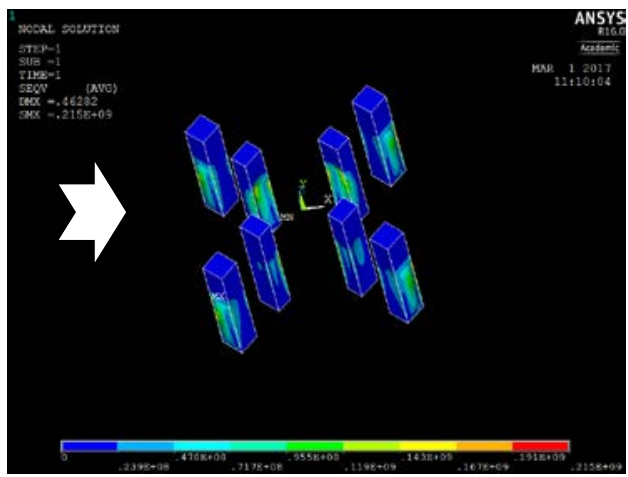

Stress effect on columns (Pa)

Figure 7 Hydrodynamic pressure

The literature extensively related wave forces, pressure, energy and deformations on offshore structures to the wave height which is two times its amplitude, as can be seen in Section 2 ([13]. An assertion of this theory was recorded when the total deformation of the hull was plotted against different wave heights ' $\mathrm{H}$ ' at $0^{0}$ wave angle as shown in Figure 8, where a linear relationship was observed. As the wave height increases, the deformations on the structure increase progressively, although this is not uniform around the structure which will be discussed later. Maximum column deformation was observed along the centre line of the $14 \mathrm{~m}$ plate, and the results for deformation were computed from that region. 
The results for hull deformations at various wave headings were computed, and a contrast for the effect of current heading on the motion response recorded in [2] was observed. Maximum hull deformation occurred between $30^{\circ}$ and $45^{\circ}$ flow incidence around the cut-water-plane.

The effect of flow angle on hull deformation was observed and recorded as in Figure 9, and a gradual increase was observed between $0^{0}$ and $45^{\circ}$. No significant increase in hull deformation between $30^{\circ}$ and $45^{\circ}$, when compared with the rapid increase in deformation recorded between $0^{0}$ and $30^{\circ}$. The increase of deformation is attributed to the reduction in motions experienced on the hull within this range of flow angle, as confirmed. This phenomenon is better explained in mechanics of statics as with reduced motion, more wave energy is transformed to strain energy of the column plates resulting in greater deformation.

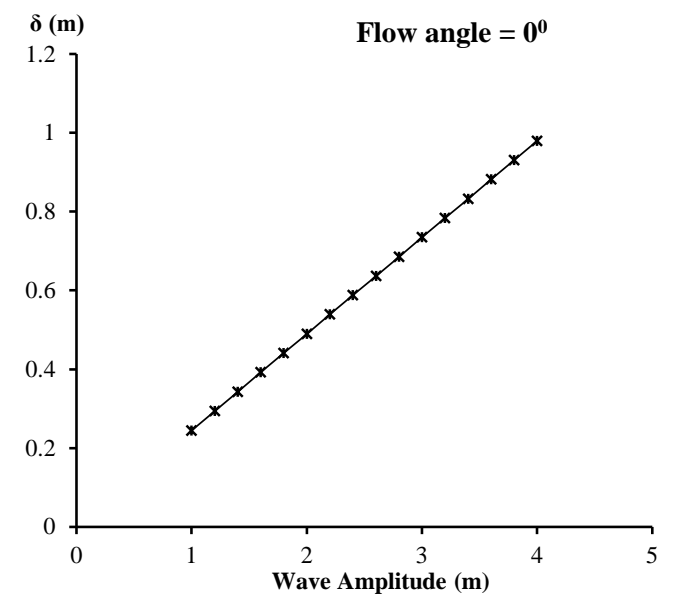

Figure 8 Relationship between wave height and maximum hull deformation

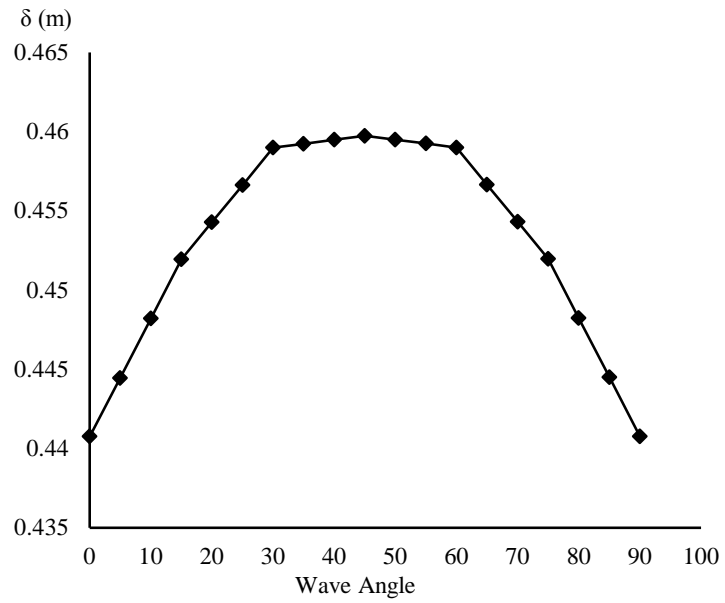

Figure 9 Relationship between wave orientation and plate deformation

Results have showed that for deep-water operating wave conditions for regular wave (small wave amplitude), the orientation have no direct effect on the maximum stress of the columns. However, as the wave amplitude increases the maximum stress of the columns gradually becomes dependent on the flow orientation, as shown in Figure 10.

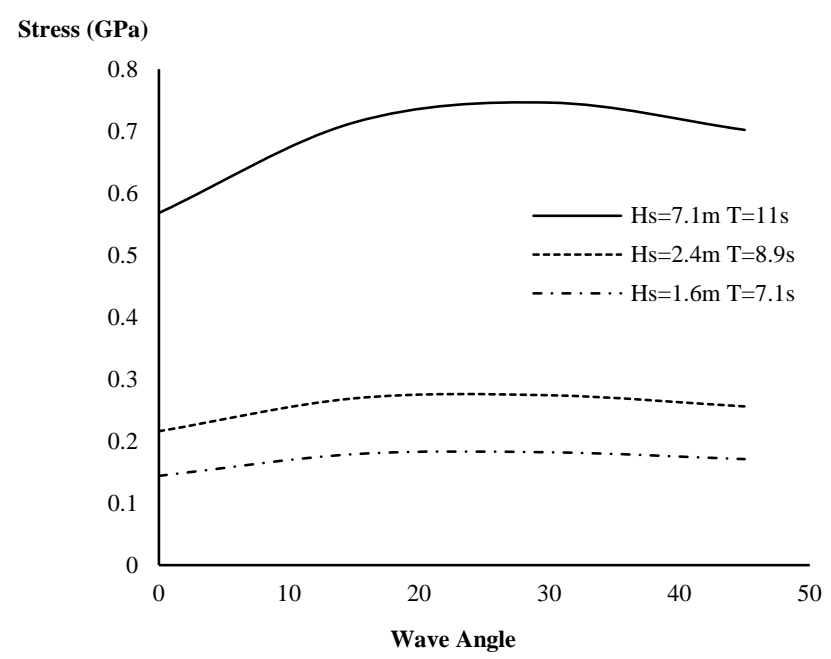

Figure 10 Relationship between wave and flow angle on column stresses 
ABS and DNV standards for which the strength of this hull formation is being assessed do not necessarily provide conditions for stress limits in relation with flow angle. Conventional design assessments are therefore carried out using maximum values.

\subsection{Column Stress Analysis}

When flow travels through a body a pool of wake builds up on the boundary layer between the flow path and the surface. As the curls gradually break away from the surface, they exert hydrodynamic drag on the surfaces situated within the pool of the wake. This drag force coupled with some additional forces exerts stress on the body.

\subsubsection{Column Area Analysis (no hull motion)}

Figure 11 shows contour plots of von Mises stress distribution around the columns with flow angles of $0^{0}$ and $45^{\circ}$ for dynamic load case with no hull motion. The stress profile around the columns was studied, and maximum stresses were located in the regions where the hull was subjected to high wave energy as shown in Figures 11. There is a high level of non-uniformity of the stress level around the inner columns, which is also proportional to the flow angle. Maximum stress was observed between $30^{\circ}$ and $45^{\circ}$. Another very useful finding from the stress analysis was the 'low-stress profile' recorded between each pair at $0^{0}$ flow angle, which gradually increases with the wave orientation. Results for straight-edge columns was considered in the study, which is the worst possible column design.

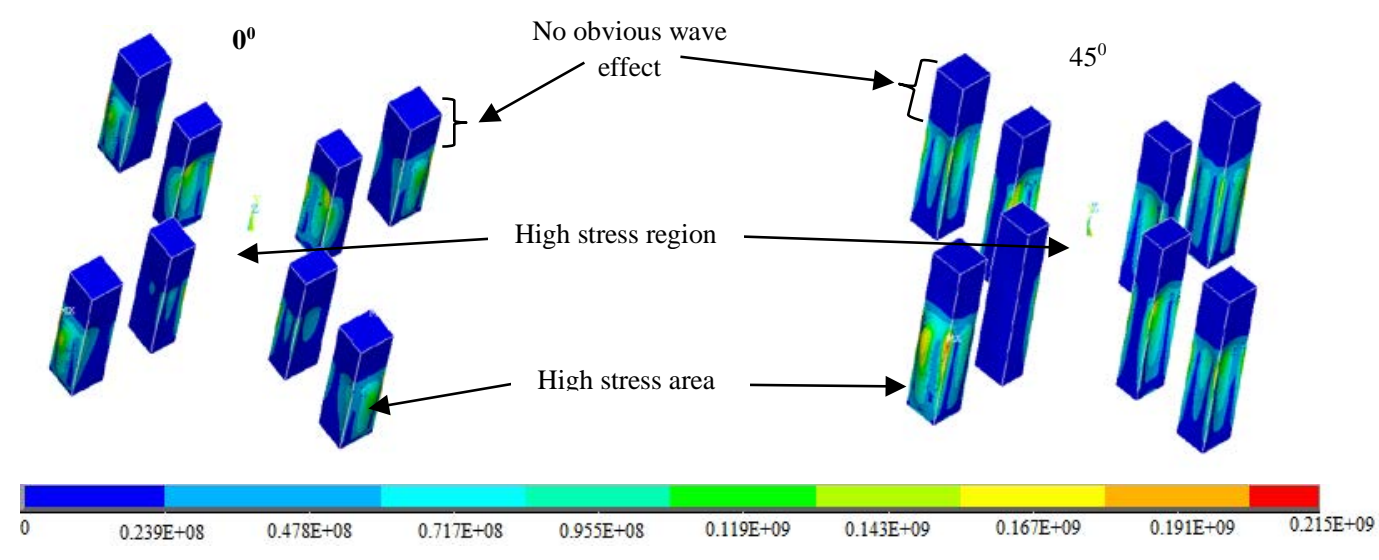

Figure 11 Column area analysis with no hull motion (Pa)

The plates exposed to the central area of the column arrangement experiences a very high level of stresses irrespective of the flow angle, as shown in Figure 11. This suggests a high level of sloshing and flow disturbance within the hull that will have a direct effect on the risers, since the drilling and production risers are housed within this area. It is also very important to mention that the unsubmerged area of the columns does not experience any significant stress concentration. The most unstable areas around the columns are the areas exposed to the region between each pair. The stress profile around this region was observed to be different on each pair, and as the angle increases the difference become more pronounced, as can be observed also in Figure 11. Reinforcing the plates around this region for both the inner and outer columns will therefore be based on the situation with the highest stress level; which was recorded around the two parallel columns at $45^{\circ}$ flow angle. The level of stress variation observed specifically around the inner columns also suggested possible instability on the entire column. A subsequent study on the buckling and failure modes is ongoing to further explain and effect of plate thickness and transverse reinforcement on the stability of each column.

\subsubsection{Topside Effect (Dynamic Load Case with Hull Motion)}


The effect of topside weight on the column's von Mises stress distribution was studied for dynamic load case. The columns were checked for possible yielding to identify areas where high reinforcement will be required. Two topside cases were checked, i.e., the PC-Semi topside (Type A) postulated by [25] and conventional semisubmersible topsides (Type B) (See Figure 12). Most operating deep-draft semisubmersibles in deep waters are designed with the Type-B topside formation (e.g. Petrobras 55). In this study, the topside weight has been considered as the summation of the mass of the topside steel and the deck mass (facilities and utilities), as presented in Table 5.
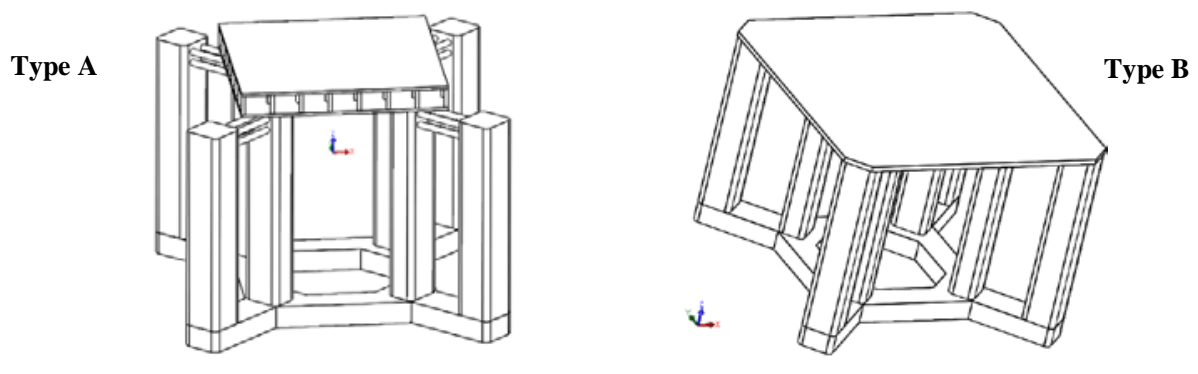

Figure 12 Topside definition

From the analyses, the following key observations were found.

o Maximum stresses were observed around the pontoon section for Type-B topside

0 Twisting and bending phenomenon are transferred from the inner columns to the outer columns for Type-A topside.

o High compressive stresses were observed on the inner columns for Type-A topside.

o From an independent column analysis (excluding all other structural members), the maximum stress was observed at the top of the inner columns for Type-A topside, and at the base of the inner columns for Type-B topside, as shown in Figure 13

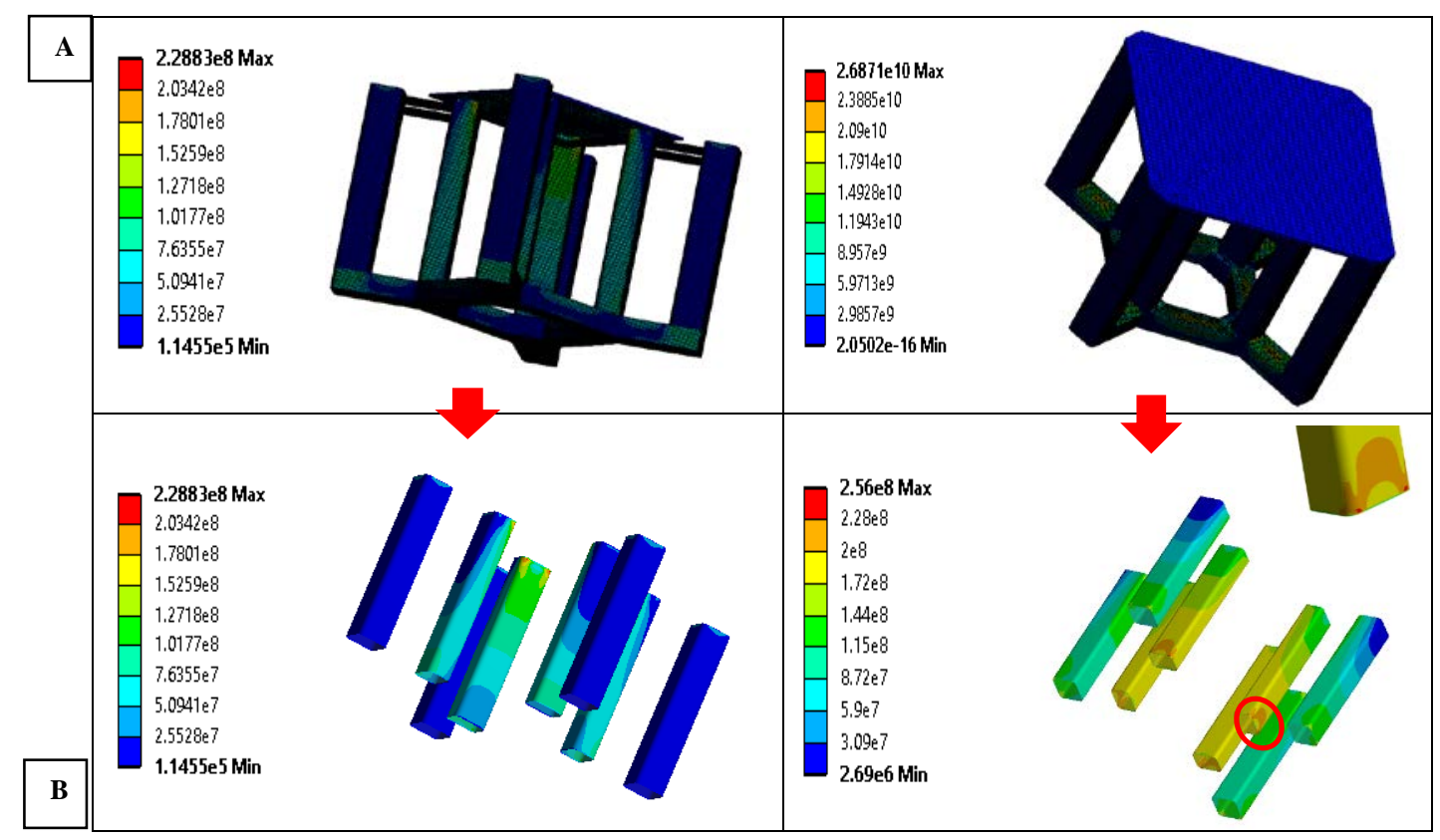

Figure 13 Topside effect on column stress distribution (Pa) 


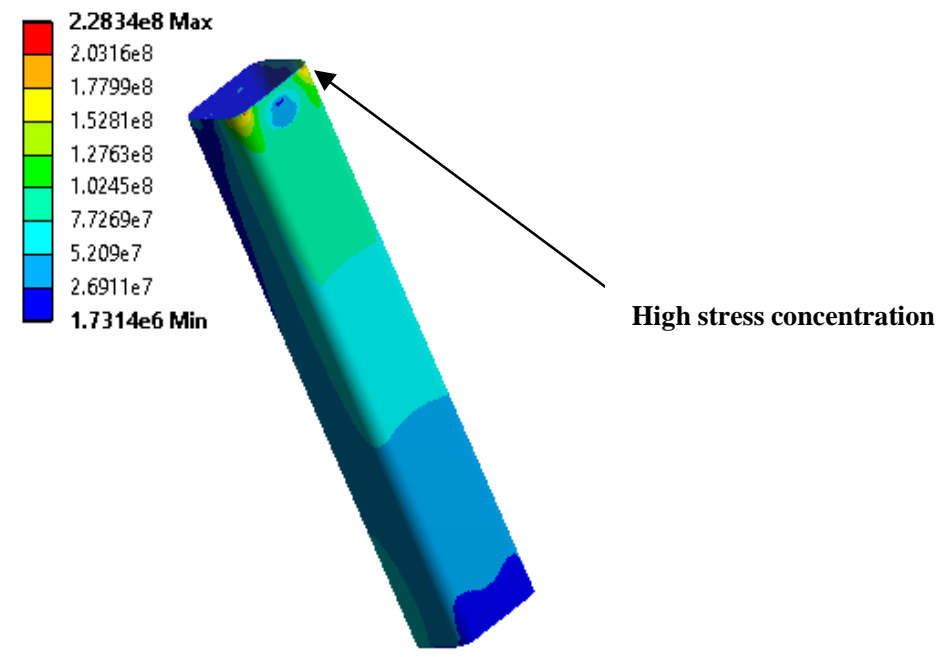

Figure 14 Inner column of Type-A topside (stress in Pa)

Figure 13 shows the results for the stress distribution on the columns for a deck mass of $30.5 \times 10^{6} \mathrm{Kg}$, considering pressure and response parameters developed from 100years wave return period. The results show significant variations between the column stress pattern for Type-A and Type-B topsides. For both cases more stresses are distributed around the inner column, but the areas of concentration are different. This indicates that the inner columns are likely to require more stiffeners and girders during scantling as compared to the outer columns, despite the fact that outer columns are bigger than the inner ones. Figure 14 shows an enlarged contour plot of one of the inner columns for Type-A topside. The results of the direct stress in the z-direction (which is not shown here) show a compressive behaviour on the column from the topside weight, and very high concentration at the edges of the connection between the topside and the column. This area will require a level of steel reinforcement in order to effectively avoid failure for high topside weight. Table 8 shows results obtained for maximum stress on columns for Type-A topside, for a deck mass range of $25.1 \times 10^{6} \mathrm{Kg}$ to $34 \mathrm{x}$ $10^{6} \mathrm{Kg}$. A steady increase was observed for both static and dynamic load cases.

Table 8 Effect of topside weight on column maximum stress (type A)

\begin{tabular}{c|c|c|c}
\hline Deck Mass & Weight & Static & Dynamic \\
\cline { 3 - 4 }$(\mathrm{x} \mathrm{10} \mathrm{Kg})$ & $(\mathrm{MN})$ & $\boldsymbol{\sigma}_{\boldsymbol{e}_{\max }}(\mathrm{MPa})$ & $\boldsymbol{\sigma}_{\boldsymbol{e}_{\max }}(\mathrm{MPa})$ \\
\hline 25.1 & 246.23 & 147.24 & 201.92 \\
26.0 & 255.06 & 153.52 & 205.50 \\
28.0 & 274.68 & 164.25 & 213.46 \\
30.0 & 294.30 & 175.98 & 221.45 \\
32.0 & 313.92 & 184.71 & 229.38 \\
34.0 & 333.54 & 191.34 & 237.32 \\
\hline
\end{tabular}




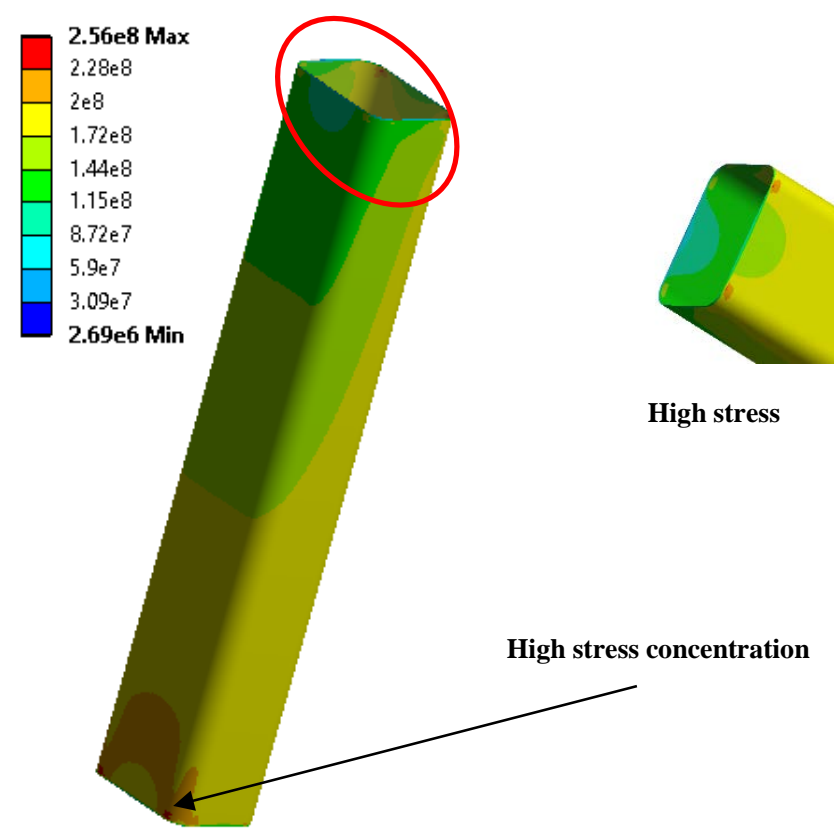

Figure 15 Type-B Inner column (stress in Pa)

For Type-B topside, high stress concentration was observed for both the topside-column connection and the column-pontoon connection areas; as presented in Figure 15. From the contour plots, the stress concentration is highest around the bottom area of the inner columns, high steel reinforcement is likely to be recommended around these areas.

The inner column braces for Type-A topside were also analysed. The stress level in the braces was much smaller than the strength of the material likely to be used (350MPa-550MPa). Figure 16 shows a representation of stresses in a brace.
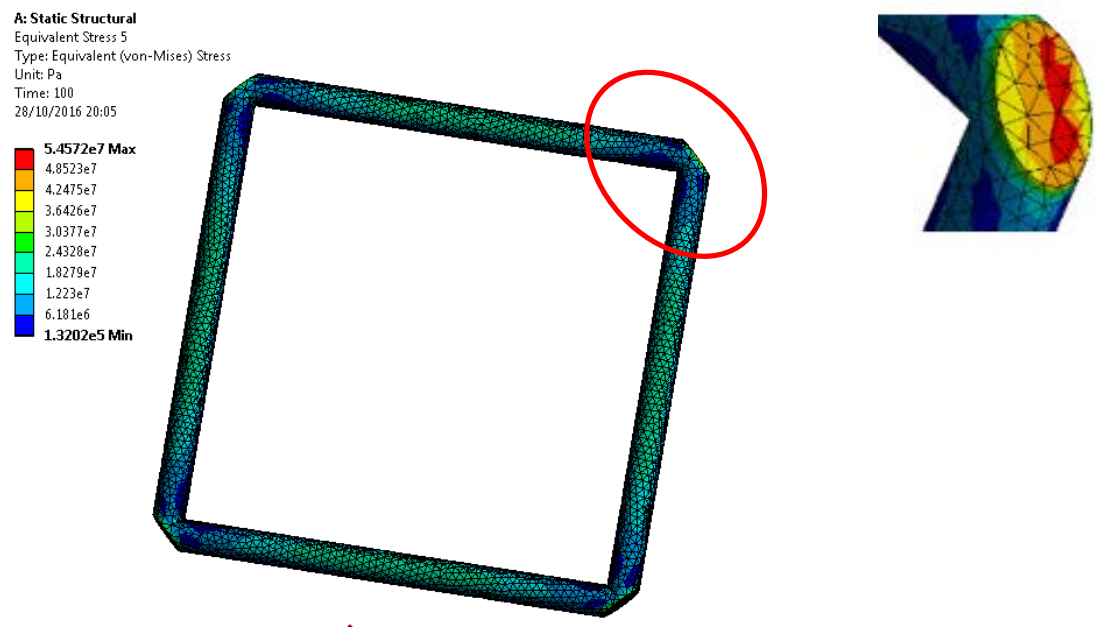

Figure 16 Stress on inner columns braces (stress in Pa)

\subsection{Analysis of Column Base for Pontoon Joint Assessment}

Sizing the column-pontoon joints has been a critical issue from the early design stages of this hull because of the slenderness ratio of the columns, and the possible stress transfer from its bending and deflection during rough weather conditions. The stress exerted on the column-pontoon joint are as a result of the deformations on the surface of each plate, the weight of the topside, and the bending experienced in the entire structure (It 
is worth mentioning that the effect of the topside weight has not be considered in this analysis). To understand this, investigations were carried out on each base edge. The length and breadth of the edges were categorized into leading edges and rear edges. The $14 \mathrm{~m}$ edges facing the flow direction are the leading lengths (LL), while the edges with $10.4 \mathrm{~m}$ and $13.4 \mathrm{~m}$ facing the flow are the leading breadths (LB) for the inner and outer columns respectively. The opposite sides are the rear length (RL) and rear breadths (RB). Results were recorded for $0^{0}$ flow angle because the flow pattern experiences the highest fluid circulation around the columns [2] [10], and the investigation is focused on understanding the effect of this phenomenon on the stress profile around the hull formation. For environmental conditions, $\mathrm{Hs}=2.1 \mathrm{~m}$ at $\mathrm{T}=7.1 \mathrm{~s}$ was selected.
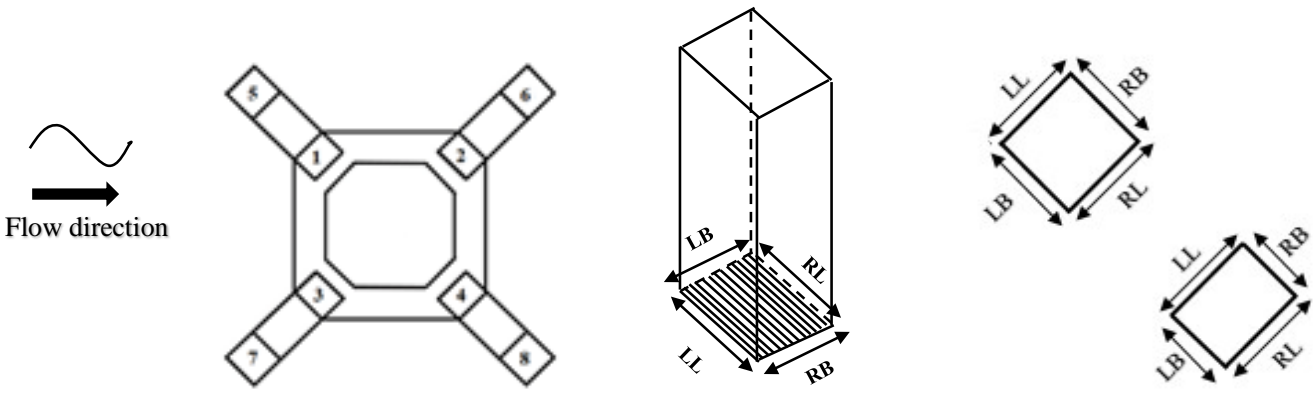

Figure 17 Base stress label

$\sigma_{l l} \quad$ Stress distribution on the leading length from the shear point

$\sigma_{l b} \quad$ Stress distribution on the leading breadth from the shear point

$\sigma_{r l} \quad$ Stress distribution on the rear length from the rear mid-point

$\sigma_{r b} \quad$ Stress distribution on the rear breadth from the rear mid-point
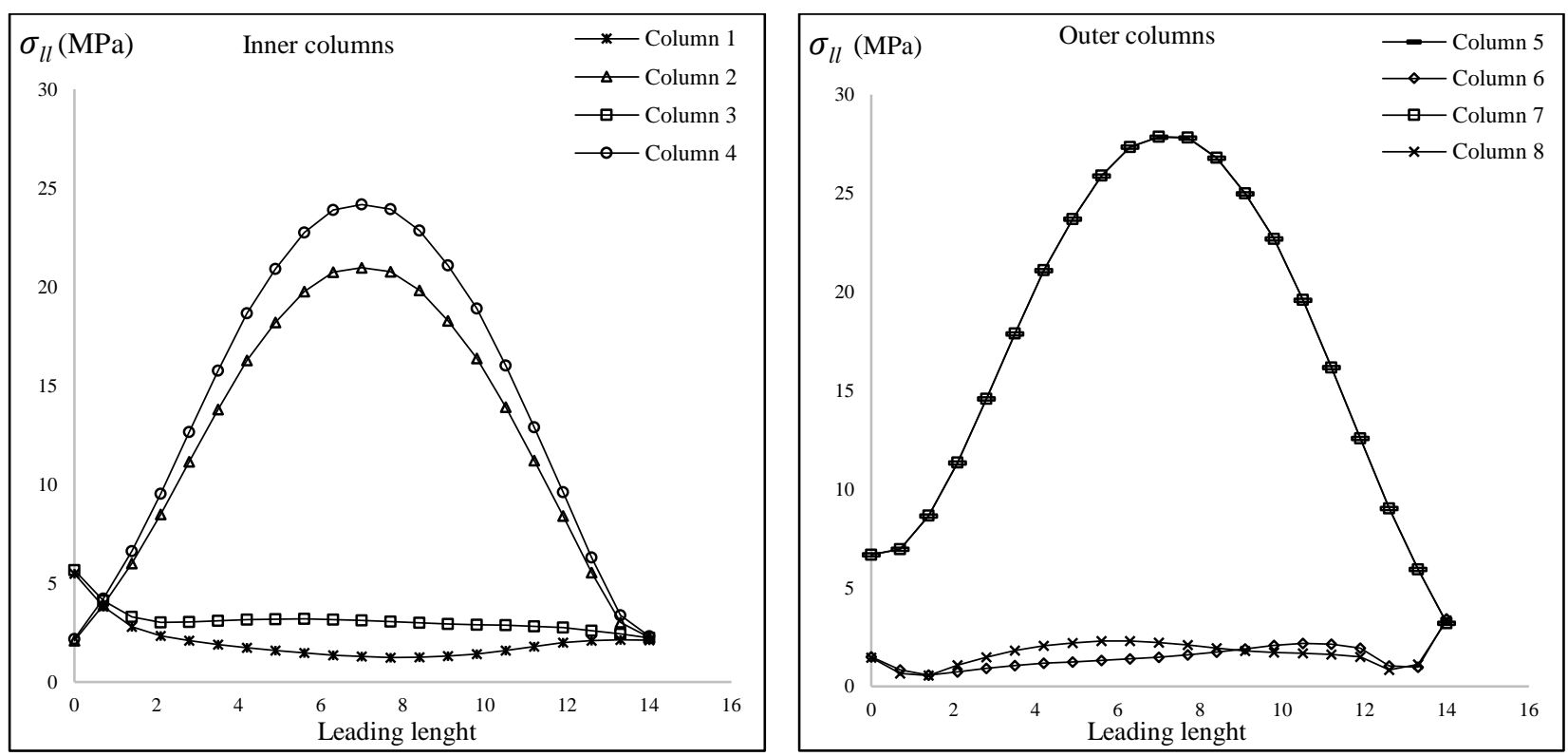

Figure 18 Stress distribution on the leading length from the shear point $\left(\sigma_{l l}\right)$

The analysis of the stress distribution on the leading length of each column is shown in Figure 18. There are similarities between the stress profile on columns 2, 4, 5 and 7, which all suggest high deflection at the centre of the $14 \mathrm{~m}$ plate generating high stress on the rigid connection joint. It is observed that the line graphs of columns 5 and 7 coincide with each other, i.e. have the same values. This was observed for all four edges 
of both columns, and will be discussed in the latter part of this section. Columns 1, 3, 6 and 8 apparently have low stress values on their leading lengths with slightly higher values at the edges where the fluid separated.

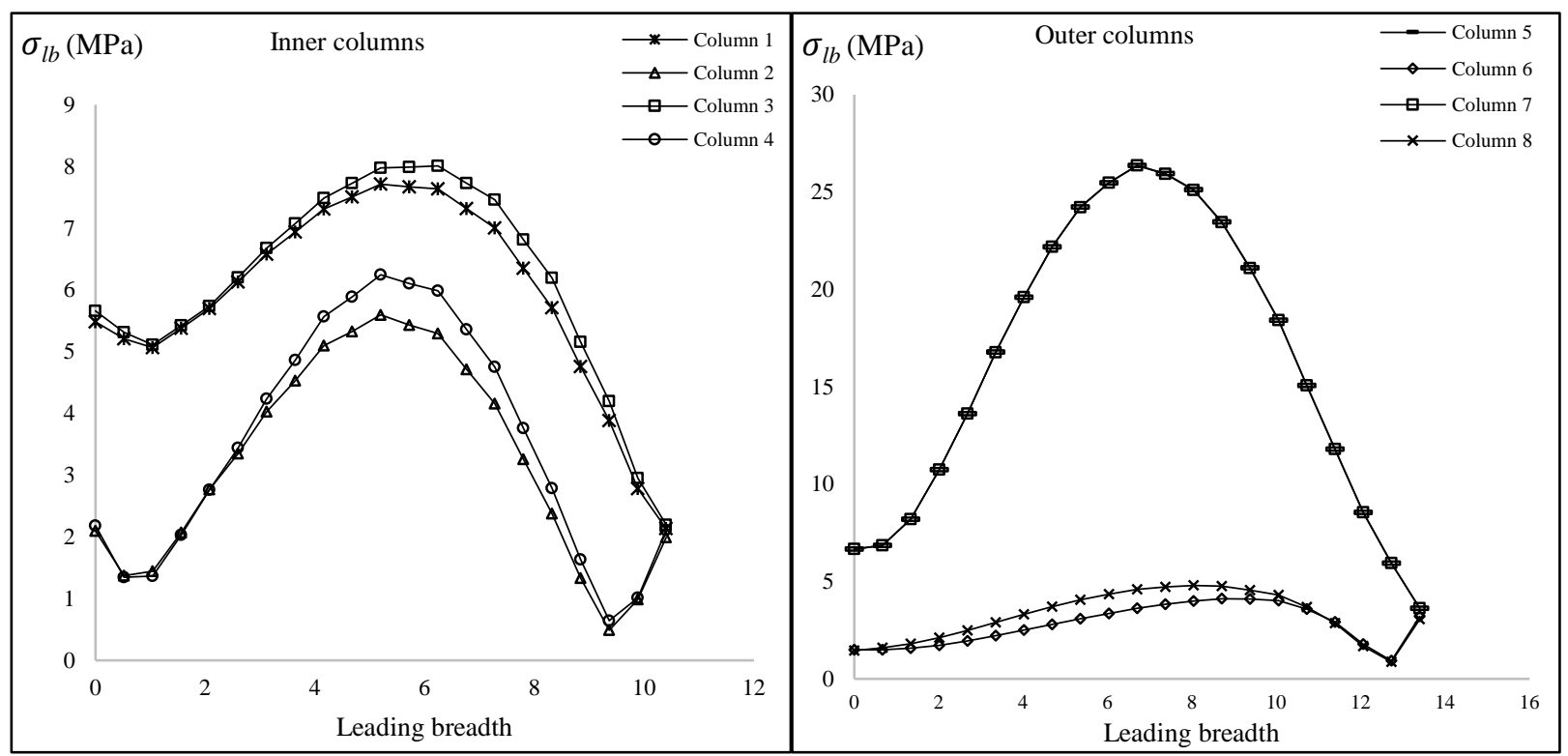

Figure 19 Stress distribution of the leading breadth from the shear point $\left(\sigma_{l b}\right)$

The breadth of the inner columns is shorter than that of the outer ones (10.5m and $13.4 \mathrm{~m}$ respectively), creating a increased stress profile for the outer columns.
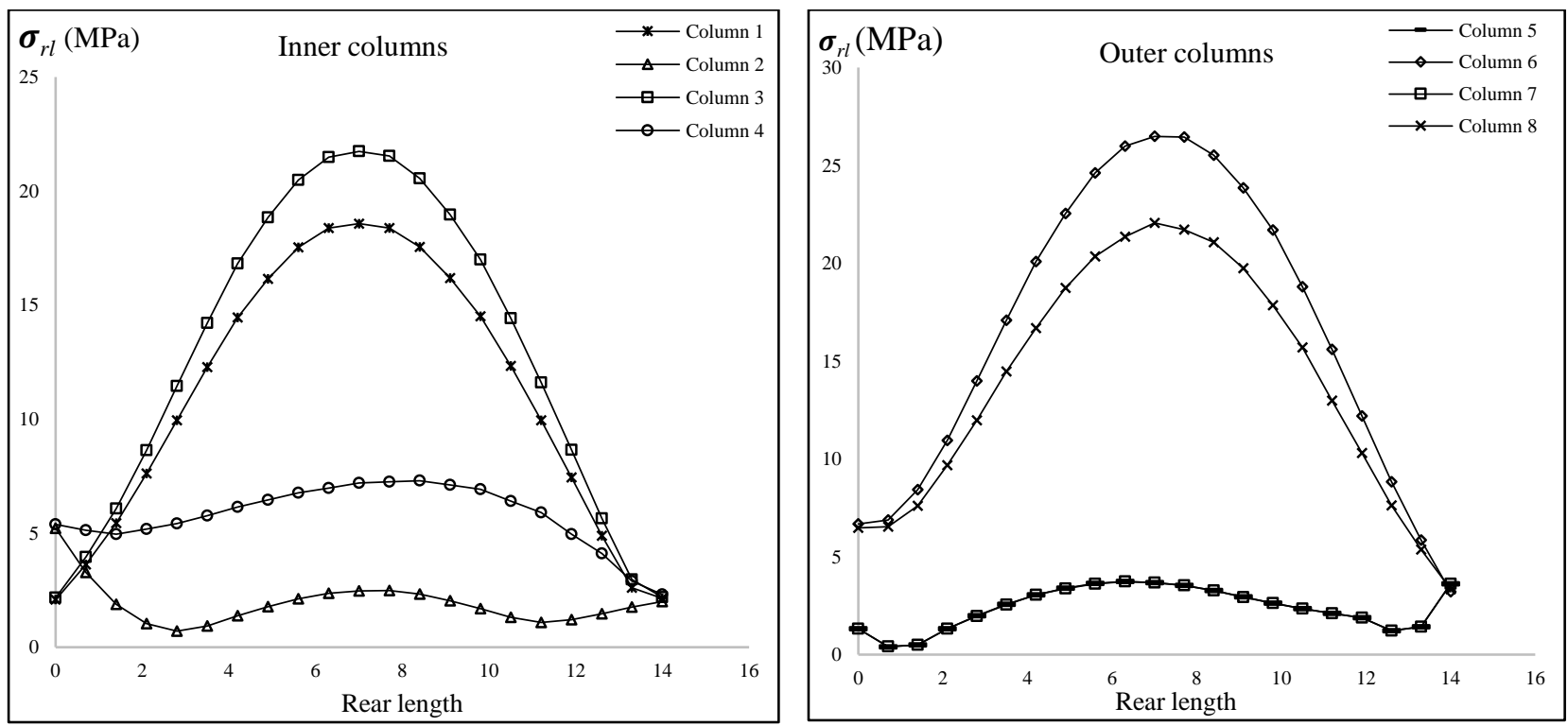

Figure 20 Stress distribution of the rear length from the rear mid-point $\left(\sigma_{r l}\right)$

The stress relationships observed for the rear lengths (see Figure 20) were quite different from what was observed earlier on the leading lengths. Figure 21 indicates an uneven stress distribution around the inner columns as mentioned earlier sited in the study. The vortex/wake formation behind the hull is responsible for the high stress observed on the rear lengths of columns 6 and 8 as they are the columns at the extreme rear end of the hull, while the contrasted was observed for columns 2, 4, 5 and 7. Figure 21 shows the corresponding stress profiles around the rear breadths, which generally have smaller stress range when compared to the rear lengths. 

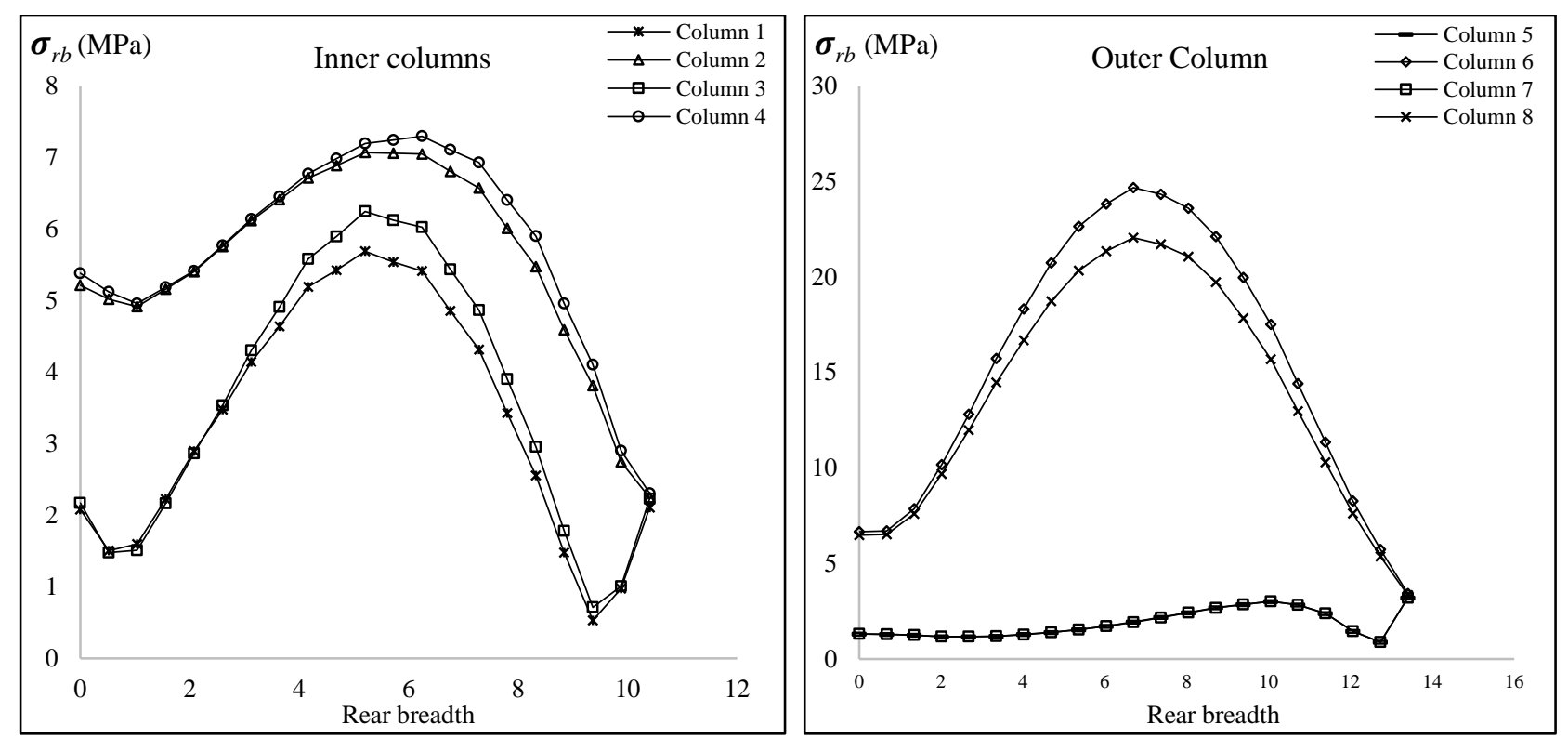

Figure 21 Stress distribution of the rear breadth from the rear mid-point $\left(\sigma_{r b}\right)$

Figures 18-21 has described the stress curves at the base of each column. This will makeup part of the criteria needed for designing and sizing the column-pontoon joint connects, which has been cited as a fundamental challenge in the design of the hull formation.

\section{Conclusion}

The structural effects of hydrodynamic loads on the columns of a paired-column semisubmersible hull formation have been studied using finite element modelling approach, and the nature of deformations and stress profiles were recorded. A series of hydrodynamic models were developed in ANSYS AQWA to calculate the responses, pressure and drag effects on and around the hull for different weather conditions. Results for hydrodynamic pressures and responses were transferred into the finite element model for structural analysis. Formation for boundary conditions was carried out using the conditions from the dynamic response of the structure of the hull and hydrostatic parameters of the hull. The hollow columns and pontoon section were modelled using shell elements, while the trusses, braces and connections were modelled using 3D solid elements. The mass distribution around the structure was calibrated / estimated considering operating semisubmersibles and the effect of the weight of the deck facilities and utilities on the stress distribution around the columns during dynamic load case was investigated.

Results from our study have suggested that the stress distribution around the columns for small amplitude wave, is likely to be independent of the flow orientation (or wave propagation direction). However, as the amplitude of the wave increases, the effect of the flow angle becomes evident on the stress distribution. It can be advised that in the process of designing this hull formation for serve storm and high wave loadings, the effect of wave orientation and wave phase has to be considered, especially when maximum stresses and deformation experienced in any of the columns are requested. The effects of small and large topsides on the stress distribution on the columns were further investigated, from which higher stress distribution were observed around the inner columns relative to the outer ones, suggesting more reinforcements are required for the inner columns. The smaller deck size was observed to have less stress level as expected, but with a unique stress concentration at the topside-column connection point. Extra steel reinforcement and weld sizing might be required around this area. 


\section{References}

[1] C. Odijie and J. Ye, "Effect of vortex induced vibration on a paired column semi-submersible platform," International Journal of Structural Stability and Dynamics, vol. 15, no. 8, p. 1540019, 2015.

[2] J. Zou, P. Poll, D. Roddier, N. Tom, and A. Peiffer., "VIM Testing of a Paired Column SemiSubmersible.," ed, 2013.

[3] J. Zou et al., "VIM Model Testing and VIM Induced Mooring Fatigue of a Dry Tree paired Column Semisubmersible Platform," ed: Offshore technology Conference, 2014.

[4] J. Zou, "VIM Response of a Dry Tree Paired-Column Semisubmersibles Platform and its Effects on Mooring Fatigue," ed: Society of Naval Architects and Marine Engineers, 2014.

[5] X. J. Chen, Y. S. Wu, W. C. Cui, and J. J. Jensen, "Review of hydroelasticity theories for global response of marine structures. ," vol. 33(3) ed: Ocean Engineering, 2006, pp. 439-457.

[6] J. N. Newman, "Wave effects on deformable bodies," Applied Ocean Research, vol. 16, no. 1, pp. 4759, 1994.

[7] M. Kashiwagi, "Hydrodynamic interactions among a great number of columns supporting a very large flexible structure," Journal of Fluids and Structures, vol. 14, no. 7, pp. 1013-1034, 2000.

[8] S. A. Sannasiraj, R. Sundaravadivelu, and V. Sundar, "Diffraction- radiation of multiple floating structures in directional waves," Ocean Engineering, vol. 28, no. 2, pp. 201-234, 2001.

[9] H. Kagemoto and D. K. P. Yue, "Interactions among multiple three- dimensional bodies in water waves: an exact algebraic method," Journal of Fluid Mechanics, vol. 166, pp. 189-209, 1986.

[10] C. Odijie and J. Ye, "Understanding Fluid-Structure Interaction for high amplitude wave loadings on a deep-draft paired column semi-submersible platform:a finite element approach," presented at the International Conference on Light Weight Design of Marine Structures, 2015.

[11] S. J. Kim, D. Spernjak, S. Holmes, V. Vinayan, and V. Antony, "Vortex- Induced Motion of Floating Strictures: CFD Sensitivity of Turbulence Model and Mesh refinement," ed: 34th International Conference on Ocean, Offshore and Arctic Engineering, 2015.

[12] RPSEA, "ULTRA-DEEPWATER DRY TREE SYSTEM FOR DRILLING AND PRODUCTION IN THE GULF OF MEXICO," Norway2014, Available: http://www.rpsea.org/media/files/project/38e0afee/101214405-02-FR-UDW Dry Tree System Drilling Production GOM-12-30-14 P.pdf.

[13] T. S. Sarpkaya, Wave Forces on Offshore Structures [electronic resource]. Cambridge : Cambridge University Press, 2010.

[14] R. Toscano and E. Dvorkin, "A shell element for finite strain analyses: hyperelastic material models," Engineering Computations, vol. 24, no. 5, pp. 514-535, 2007.

[15] E. N. Dvorkin and K. J. Bathe, "A Continuum Mechanics Based Four-Node Shell Element for General Non-Linear Analysis," ed: Department of Mechanical Engineering, Massachusetts Institute of Technology, Cambridge USA., 1983.

[16] R. D. Cook, Concepts and applications of finite element analysis, 2d ed. ed. New York : Wiley, 1981.

[17] T. R. Chandrupatla, Introduction to finite elements in engineering, 3rd ed. ed. Upper Saddle River, N.J. : Prentice Hall, 2002.

[18] T. P. Estefen and S. F. Estefen, "Buckling propagation failure in semi-submersible platform columns," Marine Structures, vol. 28, pp. 2-24, 2012.

[19] I. Senjanovic', S. Malenica, and T. e. S., "Investigation of ship hydroelasticity," Ocean Engineering, vol. 35, pp. 523-535, 2007.

[20] Ansys. (2013, 15th March). ANSYS Mechanical APDL Theory Reference (17 ed.). Available: http://148.204.81.206/Ansys/150/ANSYS\%20Mechanical\%20APDL\%20Theory\%20Reference.pdf

[21] API 2INT-MET, "Interim Guidance on Hurricane Conditions in the Gulf of Mexico," ed: American Petroleum Institute, 1997.

[22] DNV-RP-205, "Environmental Conditions and Environmental Loads," ed: Det Norske Veritas, 2007.

[23] Structural Design of Self-Elevating Units (LRFD Method), 2012.

[24] Guide for Buckling and Ultimate Strength Assessment for Offshore Structures, 2004.

[25] RPSEA, "Ultra Deepwater Dry Tree System for drilling and Production," ed: Research Partnership to Secure Energy for America, 2009. 
*Correspondence author: Jianqiao Ye (j.ye2@lancaster.ac.uk) 